Published in final edited form as:

Optom Vis Sci. 2013 November ; 90(11): 1161-1164. doi:10.1097/OPX.0000000000000117.

\title{
Myopia-Yesterday, Today, and Tomorrow
}

\author{
Donald O. Mutti, \\ Columbus, $\mathrm{OH}$ \\ Jane Gwiazda, \\ Boston, MA \\ Thomas T. Norton, \\ Birmingham, AL \\ Earl L. Smith III, \\ Houston, TX \\ Frank Schaeffel, and \\ Freiburg, Germany \\ Chi-ho To \\ Hong Kong, China
}

Ideas about the etiology of myopia used to be trapped in a debate of either/or. Early descriptions placed the blame on the environment, particularly long periods of close work. ${ }^{1}$ In the mid-20th century, Sorsby and Benjamin ${ }^{2}$ swung the pendulum strongly toward genetics when they stated flatly that "studies on twins have shown that all refractions of the eye-emmetropia, hypermetropia, and myopia—are determined genetically." Nothing ruins a good dogma like data. The more recent view, informed by an explosion of information from research in the last 20 years, has moved toward a melding of these two extremes into a richly detailed combination of both nature and nurture. The idea that refractive error was hardwired into genes was shattered in 1977 when Wiesel and Raviola ${ }^{3}$ showed that eyelid suture produced myopia in monkeys and, then again, in 1978 when Wallman and coworkers ${ }^{4}$ showed that simple frosted occluders resulted in large amounts of myopia in chicks. The myopia research community truly lost one of its pioneers when Josh Wallman passed away in 2012. These deprivation experiments were followed by lens experiments by Schaeffel and coworkers ${ }^{5}$ whose powerful paradigm has shown that the eyes of young animals of an amazing variety of species respond to the sign and the magnitude of imposed defocus by modulating their rates of axial growth to compensate for the defocus and become effectively emmetropic. The fovea was originally assumed to be the seat of this local growth regulation because of its superior acuity, but later work showed that the retinal periphery also plays a role and can actually override the fovea during compensation for blur. ${ }^{6}$ This insight has stimulated interest in specialty refractive corrections such as multifocal contact lenses and orthokeratology, interventions that provide myopic defocus to the periphery in an attempt to rein in the growth of the myopic eye.

The research on myopia has not only taken place in laboratories exploring animal models (with many of these in schools and colleges of optometry). Large-scale human studies 
conducted around the world, in the United States, the European Union, Australia, Singapore, Hong Kong, and China, have added a new perspective on the roles of both environment and heredity. Earlier emphasis on near work as the major environmental factor influencing refractive error has been joined by interest in time outdoors as a protective factor against developing myopia. ${ }^{7-9}$ The general impression that heredity was refractive error destiny has been replaced by an extensive list of specific single nucleotide polymorphisms that are associated with myopia. ${ }^{10,11}$ Their functional significance remains an intriguing mystery, although some play provocative roles in ion transport, extracellular matrix formation, retinal ganglion cell development, and early eye development.

This background on myopia research is brief because much of the progress has been recent. "Myopia" was not even a key word at ARVO until 1989. The developments outlined above form the backdrop for the 20 articles presented in this special issue of Optometry and Vision Science devoted to myopia. Contrast that with seven original articles total that were just beginning to touch on these topics in the last two special issues on myopia in Optometry and Vision Science in 2009 and 2011. The articles in this issue are specific pieces of new research, but rather than seeing them as isolated pieces of knowledge, see them as touching on the big themes above: the protective effect of time outdoors, the control of eye growth through manipulation of foveal or peripheral defocus, image quality during accommodation, retinal physiology, the genetics of scleral collagen, and insights on how the past can inform the present in myopia research.

\section{ENVIRONMENT AND REFRACTIVE ERROR-TIME OUTDOORS AND PERIPHERAL DEFOCUS}

Identification of cascades behind basic biological processes like formation of adenosine triphosphate or photosynthesis is a discovery worth more than a couple of Nobel prizes. Unraveling the signal cascade(s) regulating eye growth requires placement of the numerous substances known to affect the growth of the eye into the proper order and relationship. One of these substances is dopamine, released in response to light exposure and of prime interest as the potential basis for the protective effect on myopia of children spending more time outdoors. ${ }^{12}$ Nickla and coworkers ${ }^{13}$ noticed that inhibition of either dopamine or nitric oxide stopped its protective effects against experimental myopia. In the current study, inhibition of nitric oxide made the dopamine agonist quinpirole ineffective, suggesting that dopamine is involved earlier in the cascade and that nitric oxide is a downstream product necessary for protection against myopia. This report is timely, given the current interest in time outdoors in humans, one that will open up the conversation about the effects of light beyond dopamine. Another animal study with important human implications comes from the Smith laboratory. Previous work had shown that hemifield deprivation and hyperopic defocus accelerate local growth in the corresponding retinal area. ${ }^{14}$ The current study shows that hemifield myopic defocus in monkeys slows local growth in the corresponding retinal area. ${ }^{15}$ This finding is good news as one might predict that a full circle of peripheral myopic defocus would have a similar beneficial effect in a myopic child. A genetics-only view such as from Sorsby could hardly imagine that the shape of the eye could be distorted by local defocus, as shown by the high-resolution magnetic resonance images in this study. 


\section{CHOROIDAL RESPONSES TO DEFOCUS}

Thickening of the choroid in the chick is one of the most striking responses to the imposition of myopic defocus that slows the growth of the eye. ${ }^{16}$ Do humans retain this "inner chicken" and the ability to have the choroid participate in regulation of axial length? The research from Chakraborty and coworkers, ${ }^{17}$ including the current report, suggests the answer is yes. Previous work showed how imposed myopic defocus in mature eyes within a day thickens the choroid, shortens axial length, and shifts the daily rhythm of this process. ${ }^{17}$ The current study extends this work by showing that imposed hyperopic defocus accentuates the thinning of the choroid and lengthening of the axial eye without a disruption in rhythm. ${ }^{18}$ Work from Lan and colleagues ${ }^{19}$ brings the themes of light exposure and choroidal changes together. A period of bright light exposure thickened the choroids of chicks later in the day compared with those of control chicks exposed to normal indoor illumination. For 5 days, control chicks had thinner choroids compared with baseline, but bright light exposure maintained choroidal thickness over time. ${ }^{19}$

\section{PERIPHERAL DEFOCUS AND MYOPIA CONTROL}

We still await the results of the definitive multiyear, randomized, clinical trial of peripheral optical treatment for controlling the progression of myopia in children. In the meantime, Walline and colleagues ${ }^{20}$ examined myopic children in bifocal soft contact lenses during a 2 -year period compared with historic controls wearing single-vision contact lenses. The 27 children who completed 2 years progressed by about -0.50 diopters compared with the control children's progression of about -1.00 diopter. The most important finding was that, unlike other myopia treatments, the benefits did not seem confined to only the first year of treatment. Berntsen and Kramer ${ }^{21}$ examined the optics of center distance soft multifocal contact lenses. The added value of this work is that the peripheral optical profile for nonpresbyopic wearers was measured both at distance and at near. These designs would be less interesting if young wearers used the add power to avoid accommodation, thereby experiencing substantial amounts of foveal hyperopic defocus at near from the central zone. The young adults did not show any more lag than normal myopic children. They preferred their own accommodation to using the add power of the contact lens. Only one design was evaluated (Biofinity Multifocal D), but these bifocal lenses produced absolute myopia in the periphery at distance and produced emmetropia at several peripheral angles at near.

When it comes to supplying myopic peripheral defocus, overnight orthokeratology is still the champion. The main results from the Myopia Control with Orthokeratology contact lenses in Spain study have been reported elsewhere $(0.22 \mathrm{~mm}$ less axial elongation in 2 years of treatment), ${ }^{22}$ but a follow-up report sought to ask what factors in a patient made for a better response. The authors identified a long list of positive factors for a better effect from overnight orthokeratology that needs to be evaluated prospectively (children who were older, had earlier onset of myopia, female sex, lower rate of myopia progression before baseline, less myopia at baseline, longer anterior chamber depth, greater corneal power, more prolate corneal shape, larger iris diameter, larger pupil sizes, and lower levels of parental myopia). ${ }^{23}$ Kang et al. ${ }^{24}$ from the Swarbrick laboratory measured peripheral refraction profiles after 2 weeks of orthokeratology lens wear, data that make for a nice 
comparison with those in the Berntsen article. In addition, Kang et al. ${ }^{24}$ attempted to manipulate fitting parameters and found that changes to optic zone diameter and peripheral curves produced no significant difference in the peripheral refraction profiles after orthokeratology. Maseedupally et al. ${ }^{25}$ made a detailed analysis of the changes in tangential curvature after orthokeratology and found nasal-temporal asymmetry with greater flattening temporally in the central cornea ( $5 \mathrm{~mm}$ diameter) and greater steepening temporally in the more peripheral zones. The specifics of these peripheral refraction profiles, including symmetry, have to be considered when future trials relate the degree of effectiveness of an optical treatment with the quality of the peripheral image being delivered.

Some corneal asymmetry may be natural and associated with emmetropia. Leung and coworkers ${ }^{26}$ made Pentacam measurements on 100 Chinese subjects across a range of ages with either compound myopic astigmatism or emmetropia. Corneas became less prolate with age in both refractive error groups. The most consistent differences from emmetropes were that myopic astigmats had more prolate temporal corneas, a shape associated with more astigmatism, at all ages and less prolate inferior corneas in the youngest two age groups. These differences may have implications for how the shape of the retinal contour develops in response to natural peripheral defocus or during an optical treatment for myopia. The relationship between corneal shape and astigmatism is an important perspective considering the large longitudinal study done on Native American children by Twelker et al. ${ }^{27}$ that showed that higher levels of astigmatism in young children were more likely to predispose them to become myopic by age 18 years.

\section{FOVEAL AND PERIPHERAL OPTICAL TREATMENTS FOR MYOPIA CONTROL}

Following the work of Smith and others regarding the ability of the retinal periphery to regulate the rate of axial growth, the usual optical strategy would be to provide relative myopic defocus to the retinal periphery as a "stop" signal. Price and coworkers ${ }^{28}$ in the Cambridge Anti-Myopia Study used a different strategy to improve accommodative response and reduce lag-reduction in positive spherical aberration — the opposite to the peripheral myopic defocus strategy of increasing positive spherical aberration. A subgroup also received accommodative facility flipper exercises. Interestingly and in contrast to the positive spherical aberration strategies of Walline et al. ${ }^{20}$ and Santodomingo-Rubido et al., ${ }^{23}$ the intervention did not alter the rate of progression of myopia. ${ }^{29} \mathrm{~A}$ higher AC/A ratio, rather than greater lag, was the variable associated with the rate of myopic progression.

Not all alterations to spherical aberration are created equal, argues Thibos and coworkers. ${ }^{30}$ The authors' optical models show how image contrast is better in the presence of hyperopic defocus when spherical aberration is positive. They theorize that negative spherical aberration, particularly during near work, in conjunction with hyperopic defocus might be an underappreciated source of image degradation. The article makes a good case for measurement of baseline spherical aberration at distance and at near during trials of optical treatments for myopia control. The Thibos laboratory goes on to argue that not all retinas are created equal. Combining factors of accommodative lag and image quality, Sreenivasan et al. ${ }^{31}$ showed that myopes had more accommodative lag and poorer image quality across 
tasks but similar levels of visual acuity compared with emmetropes. Questions remain regarding whether the myopes are simply poorer at accommodation, ${ }^{32,33}$ whether their retinal processing squeezes more information out of the retinal image than emmetropes, and whether this more effective processing constitutes a blur tolerance that promotes hyperopic defocus. The retinal periphery seems to appreciate the improvement in image quality that comes from an appropriate peripheral correction. An on-axis correction degraded peripheral grating acuity and contrast thresholds compared with what was obtained while wearing an optimal peripheral refractive correction. ${ }^{34}$ Peripheral visual performance could be restored with custom design spectacles in this study, at least across the horizontal meridian.

Overnight orthokeratology and bifocal soft contact lenses are the two main modes of altering peripheral defocus; spectacle designs have had only moderate success. ${ }^{35}$ It will be interesting to see how spectacle designs keep up with what can be delivered optically at the cornea.

\section{WHAT ABOUT THE REST OF THE EYE?}

Myopia research tends to be very axial length centric. Bailey and others ${ }^{36,37}$ have done a good job pointing out that other parts of the eye such as the ciliary muscle may be involved in myopia. Kuchem et al. ${ }^{38}$ from the Bailey laboratory extended this observation into anisometropia. Myopic eyes followed previous results, namely, that the ciliary muscle is thicker in myopes, particularly its posterior portion, but the more myopic eye in anisometropia was no different from the fellow eye. The authors argue that influences from the ciliary muscle may be just one of several pathways to myopia. More in line with the axial nature of myopia, Feng et al. ${ }^{39}$ conducted a meta-analysis of five previous studies looking at whether a variant in the lumican gene conferred a higher risk of high myopia in Chinese samples. The authors ${ }^{39}$ concluded the answer was yes with a relative risk of 1.46 for the homozygous genotype. High myopia continues to show different genetic variants compared with lower forms as this lumican gene variant was not listed in two recent and extremely large genome-wide analyses. ${ }^{10,11}$

\section{NO SUBSTITUTES FOR PATIENT-BASED DATA}

Much can be learned from the laboratory, but sometimes, there is no other way to address a question than by the hard labor of accumulating a large data set during a long period. Hyrnchak and colleagues ${ }^{40}$ at the University of Waterloo collected refractive error data on all patients of all ages seen at their clinic in 2007. The result is a wonderful figure depicting the increase in the prevalence of myopia in the young, followed by the increase in the prevalence of hyperopia and decrease in the prevalence of myopia after age $50 .{ }^{40}$ The exact numbers may be in question because the data are from a clinical sample rather than a population-based one, but the general trends have been borne out in longitudinal data sets over shorter spans of ages. The authors also noted an increase in the prevalence of myopia when their data were compared with three older data sets, consistent with reports from Asia and from the United States. Rozema and colleagues ${ }^{41}$ polished up some of these large venerable data sets for others, like the Waterloo group, to use in the future. Four "golden oldies" from Stenström, Sorsby, Zeeman, and Awerbach have been adjusted for refractive 
index and optical calculation errors and then made available as supplemental digital content in Optometry and Vision Science. ${ }^{41}$

This feature issue represents a diverse collection of work that follows the many threads that make up modern myopia research. These studies will stimulate the research that addresses the questions for tomorrow. Can peripheral defocus or time outdoors be harnessed to provide clinical benefit? What would be the results of a full-scale clinical trial of these interventions? How would one optimize growth signals if trials show good results? Do the choroid and the ciliary muscle hold new insights into axial growth and myopia? Are differences in time outdoors the reason for increasing rates of myopia across generations? To paraphrase, there are more things in heaven and earth, Kepler and Sorsby, than are dreamt of in the either/or of nature versus nurture.

\section{References}

1. Mark HH. Johannes Kepler on the eye and vision. Am J Ophthalmol. 1971; 72:869-78. [PubMed: 4940979]

2. Sorsby A, Benjamin B. Modes of inheritance of errors of refraction. J Med Genet. 1973; 10:161-4. [PubMed: 4714583]

3. Wiesel TN, Raviola E. Myopia and eye enlargement after neonatal lid fusion in monkeys. Nature. 1977; 266:66-8. [PubMed: 402582]

4. Wallman J, Turkel J, Trachtman J. Extreme myopia produced by modest changes in early visual experience. Science. 1978; 201:1249-51. [PubMed: 694514]

5. Schaeffel F, Glasser A, Howland HC. Accommodation, refractive error, and eye growth in chickens. Vision Res. 1988; 28:639-57. [PubMed: 3195068]

6. Smith EL 3rd. Prentice Award Lecture 2010: A case for peripheral optical treatment strategies for myopia. Optom Vis Sci. 2011; 88:1029-44. [PubMed: 21747306]

7. Jones LA, Sinnott LT, Mutti DO, Mitchell GL, Moeschberger ML, Zadnik K. Parental history of myopia, sports and outdoor activities, and future myopia. Invest Ophthalmol Vis Sci. 2007; 48:3524-32. [PubMed: 17652719]

8. Rose KA, Morgan IG, Ip J, Kifley A, Huynh S, Smith W, Mitchell P. Outdoor activity reduces the prevalence of myopia in children. Ophthalmology. 2008; 115:1279-85. [PubMed: 18294691]

9. Deng L, Gwiazda J, Thorn F. Children's refractions and visual activities in the school year and summer. Optom Vis Sci. 2010; 87:406-13. [PubMed: 20375747]

10. Kiefer AK, Tung JY, Do CB, Hinds DA, Mountain JL, Francke U, Eriksson N. Genome-wide analysis points to roles for extracellular matrix remodeling, the visual cycle, and neuronal development in myopia. PLoS Genet. 2013; 9:e1003299. [PubMed: 23468642]

11. Verhoeven VJ, Hysi PG, Wojciechowski R, Fan Q, Guggenheim JA, Hohn R, MacGregor S, Hewitt AW, Nag A, Cheng CY, et al. Genome-wide meta-analyses of multiancestry cohorts identify multiple new susceptibility loci for refractive error and myopia. Nat Genet. 2013; 45:3148. [PubMed: 23396134]

12. Ashby RS, Schaeffel F. The effect of bright light on lens compensation in chicks. Invest Ophthalmol Vis Sci. 2010; 51:5247-53. [PubMed: 20445123]

13. Nickla DL, Lee L, Totonelly K. Nitric oxide synthase inhibitors prevent the growth-inhibiting effects of quinpirole. Optom Vis Sci. 2013; 90:1167-75. [PubMed: 24061155]

14. Smith EL 3rd, Hung LF, Huang J, Blasdel TL, Humbird TL, Bockhorst KH. Effects of optical defocus on refractive development in monkeys: evidence for local, regionally selective mechanisms. Invest Ophthalmol Vis Sci. 2010; 51:3864-73. [PubMed: 20220051]

15. Smith EL 3rd, Hung LF, Huang J, Arumugam B. Effects of local myopic defocus on refractive development in monkeys. Optom Vis Sci. 2013; 90:1176-86. [PubMed: 24061154] 
16. Wildsoet C, Wallman J. Choroidal and scleral mechanisms of compensation for spectacle lenses in chicks. Vision Res. 1995; 35:1175-94. [PubMed: 7610579]

17. Chakraborty R, Read SA, Collins MJ. Monocular myopic defocus and daily changes in axial length and choroidal thickness of human eyes. Exp Eye Res. 2012; 103:47-54. [PubMed: 22971342]

18. Chakraborty R, Read SA, Collins MJ. Hyperopic defocus and diurnal changes in human choroid and axial length. Optom Vis Sci. 2013; 90:1187-98. [PubMed: 24061153]

19. Lan W, Feldkaemper M, Schaeffel F. Bright light induces choroidal thickening in chickens. Optom Vis Sci. 2013; 90:1199-1206. [PubMed: 24076544]

20. Walline JJ, Greiner KL, McVey ME, Jones-Jordan LA. Multifocal contact lens myopia control. Optom Vis Sci. 2013; 90:1207-14. [PubMed: 24061152]

21. Berntsen DA, Kramer CE. Peripheral defocus with spherical and multifocal soft contact lenses. Optom Vis Sci. 2013; 90:1215-24. [PubMed: 24076542]

22. Santodomingo-Rubido J, Villa-Collar C, Gilmartin B, Gutierrez-Ortega R. Myopia control with orthokeratology contact lenses in Spain: refractive and biometric changes. Invest Ophthalmol Vis Sci. 2012; 53:5060-5. [PubMed: 22729437]

23. Santodomingo-Rubido J, Villa-Collar C, Gilmartin B, Gutiérrez-Ortega R. Factors preventing myopia progression with orthokeratology correction. Optom Vis Sci. 2013; 90:1225-36. [PubMed: 24037063]

24. Kang P, Gifford P, Swarbrick H. Can manipulation of orthokeratology lens parameters modify peripheral refraction? Optom Vis Sci. 2013; 90:1237-48. [PubMed: 24076541]

25. Maseedupally V, Gifford P, Lum E, Swarbrick H. Central and paracentral corneal curvature changes during orthokeratology. Optom Vis Sci. 2013; 90:1249-58. [PubMed: 24037062]

26. Leung TW, Lam AK, Kee CS. Corneal shapes of Chinese emmetropes and myopic astigmats aged 10-45 years. Optom Vis Sci. 2013; 90:1259-66. [PubMed: 24076543]

27. Twelker JD, Miller JM, Sherrill DL, Harvey EM. Astigmatism and myopia in Tohono O'odham Native American children. Optom Vis Sci. 2013; 90:1267-73. [PubMed: 24100480]

28. Price H, Allen PM, Radhakrishnan H, Calver R, Rae S, Theagarayan B, et al. The Cambridge Antimyopia Study: variables associated with myopia progression. Optom Vis Sci. 2013; 90:1274-83. [PubMed: 24100478]

29. Allen PM, Radhakrishnan H, Price H, Rae S, Theagarayan B, Calver RI, Sailoganathan A, Latham K, O'Leary DJ. A randomised clinical trial to assess the effect of a dual treatment on myopia progression: the Cambridge Anti-Myopia Study. Ophthal Physiol Opt. 2013; 33:267-76.

30. Thibos LN, Bradley A, Liu T, López-Gil N. Spherical aberration and the sign of defocus. Optom Vis Sci. 2013; 90:1284-92. [PubMed: 24037061]

31. Sreenivasan V, Aslakson E, Kornaus A, Thibos LN. Retinal image quality during accommodation in adult myopic eyes. Optom Vis Sci. 2013; 90:1293-1304.

32. Gwiazda J, Thorn F, Bauer J, Held R. Myopic children show insufficient accommodative response to blur. Invest Ophthalmol Vis Sci. 1993; 34:690-4. [PubMed: 8449687]

33. Mutti DO, Jones LA, Moeschberger ML, Zadnik K. AC/A ratio, age, and refractive error in children. Invest Ophthalmol Vis Sci. 2000; 41:2469-78. [PubMed: 10937556]

34. Atchison DA, Mathur A, Varnas SR. Visual performance with lenses correcting peripheral refractive errors. Optom Vis Sci. 2013; 90:1305-12.

35. Sankaridurg P, Donovan L, Varnas S, Ho A, Chen X, Martinez A, Fisher S, Lin Z, Smith EL 3rd, Ge J, Holden B. Spectacle lenses designed to reduce progression of myopia: 12-month results. Optom Vis Sci. 2010; 87:631-41. [PubMed: 20622703]

36. Bailey MD, Sinnott LT, Mutti DO. Ciliary body thickness and refractive error in children. Invest Ophthalmol Vis Sci. 2008; 49:4353-60. [PubMed: 18566470]

37. Buckhurst H, Gilmartin B, Cubbidge RP, Nagra M, Logan NS. Ocular biometric correlates of ciliary muscle thickness in human myopia. Ophthal Physiol Opt. 2013; 33:294-304.

38. Kuchem MK, Sinnott LT, Kao CY, Bailey MD. Ciliary muscle thickness in anisometropia. Optom Vis Sci. 2013; 90:1313-21.

39. Feng YF, Zhang YL, Zha Y, Huang JH, Cai JQ. Association of lumican gene polymorphism with high myopia: a meta-analysis. Optom Vis Sci. 2013; 90:1322-7. 
40. Hrynchak PK, Mittelstaedt A, Machan CM, Bunn C, Irving EL. Increase in myopia prevalence in clinic-based populations across a century. Optom Vis Sci. 2013; 90:1332-42.

41. Rozema JJ, Lechat M, Tassignon MJ. Reconstruction and correction of four historical biometry data sets. Optom Vis Sci. 2013; 90:1343-50. 